\title{
Industry news: the additive manufacturing of nerve conduits for the treatment of peripheral nerve injury
}

\author{
Ying $\operatorname{Han}^{1,2} \cdot$ Jun $\operatorname{Yin}^{1,2}$
}

Received: 8 August 2021 / Accepted: 20 August 2021 / Published online: 18 September 2021

(c) Zhejiang University Press 2021

\section{Additive manufacturing (3D printing) allows for the precise spatial deposition of bi- ological and biochemical materials, even living cells to fabricate nerve conduits, which are the promising treatment for the peripheral nerve injury.}

\section{Peripheral nerve injury and nerve conduit manufacturing}

The global nerve injury repair and regeneration market is expected to reach $\$ 9.7$ billion by 2025 , a compound annual growth rate of $9.1 \%$ between 2020 and 2025 [1]. One component alone, peripheral nerve injury (PNI), encompasses 5 million new cases worldwide every year [2]. Although nerve autograft is considered the gold standard for PNI repair, this process suffers because of a short supply of autologous nerves as well as potential harm caused to the donor. In recent decades, as a promising alternative to autografts, nerve conduits have attracted significant attention, and the design and manufacturing of nerve conduits are the cutting edge of the treatment of PNI [3]. A number of commercially available nerve conduits are regulated by the US Food and Drug Administration (FDA) including NeuraGen ${ }^{\circledR}$, NeuroMatrix $^{\mathrm{TM}}$, Neurolac ${ }^{\circledR}$, Neuroflex $^{\mathrm{TM}}$, Reaxon ${ }^{\circledR}$, Nerbridge $^{\circledR}$, and Avance ${ }^{\circledR}$ [4]. These commercial nerve conduits are made mainly of biodegradable polymers, i.e., type I collagen, polysaccharides, polyglycolic acid

\section{Jun Yin}

junyin@zju.edu.cn

1 The State Key Laboratory of Fluid Power and Mechatronic Systems, School of Mechanical Engineering, Zhejiang University, Hangzhou 310028, China

2 Key Laboratory of 3D Printing Process and Equipment of Zhejiang Province, School of Mechanical Engineering, Zhejiang University, Hangzhou 310028, China
(PGA), polycaprolactone (PCL), and porcine small intestine submucosa. The bulk of these commercial nerve conduits are molded, electrospun or woven into hollow tubular constructs but lack the necessary biophysical and biochemical cues for nerve regeneration. This significantly limits their repair efficiency and capacity for connecting larger gaps greater than $3 \mathrm{~mm}$. In addition, traditional manufacturing methods used for nerve conduits, including film rolling, injection molding, electrospinning, dip coating, and coaxial extrusion, cannot meet the diverse requirements of nerve conduits in the repair of PNI.

One option, the advanced additive manufacturing method (3D printing), allows for the layer-by-layer precise spatial deposition of biological and biochemical materials, even living cells to fabricate 3D structures. Among different $3 \mathrm{D}$ printing techniques, inkjet, extrusion-based 3D printing, and vat photopolymerization are commonly used for nerve conduit fabrication to mimic the topological shape, biocompatibility, and mechanical properties of nerve tissue extracellular matrix (ECM).

\section{Recent progress in nerve conduit 3D printing}

Radulescu et al. [5] used inkjet printing to manufacture nerve conduits. In this approach, droplets of polylactic acid (PLA)/polycaprolactone (PCL) copolymer were dispensed on a rotating rod to form tubes. However, although this study was able to show that PLA/PCL nerve conduits promoted 
the attachment and outgrowth of human embryonic kidney cells, no further in vivo experiments were performed. In subsequent work, Johnson et al. [6] combined 3D scanning and extrusion-based 3D printing in order to fabricate conduits with biomimetic nerve regeneration pathways. In this approach, silicone was deposited as the support material and gelatin methacrylate (GelMA) containing either nerve growth factor (NGF) as the sensory path cue or glial cell line-derived neurotrophic factor (GDNF) as the motor path cue was dripped along the inner silicone wall serving as the luminal supplement. Additional in vivo studies were able to demonstrate the successful regeneration of bifurcated injuries across a $10 \mathrm{~mm}$ complex nerve gap in rats. In similar work, Singh et al. [7] used stereolithography (SLA) and cryogelation technology to fabricate biomimetic nerve conduits with one aligned cryomatrix lumen or four channels by using photocrosslinkable PCL. In this work, a $15 \mathrm{~mm}$ sciatic nerve defect was bridged using PCL nerve conduit indicating that nerve conduits filled with aligned cryomatrix and combined with NGF could better enhance overall regenerated nerve physiology and mimic the cellular aspects of regeneration. Zhu et al. [8] later used digital light processing (DLP) technology to manufacture nerve conduits with different geometrical features including hollow conduits, conduits with microchannels or branches, and an anatomically sized biomimetic conduit. A GelMA and poly (ethylene glycol) diacrylate (PEGDA) composite was then chosen as the photopolymerizable biomaterial. Regeneration of $4 \mathrm{~mm}$ mouse sciatic nerve gaps bridged by conduits with four channels was able to fully demonstrate the advantage of DLP printed nerve conduits.

Indeed, compared to polymeric biomaterials, bioinks containing biochemical cues or living cells enable continued nutrient supply and act to accelerate the regeneration of nerve tissues, especially for large nerve injury defects. Tao et al. [9] used a continuous DLP process to fabricate nanoparticle-inhydrogel nerve conduits; in this study, GelMA hydrogel was mixed with drug-loaded poly(ethylene glycol)-poly(3-caprolactone) (MPEG-PCL) nanoparticles to release XMU-MP-1 to increase peripheral myelination and functional recovery in rats with $10 \mathrm{~mm}$ nerve defects. In similar work, Fang et al. [10] prepared nerve conduits with the DLP method using a nanocomposite composed of reduced graphene oxide nanosheets. Thus, under a high-frequency magnetic field, carbon porous nanocookies facilitated magnetoelectric conversion for the release of growth factors and cell stimulation. The effects of repairing $10 \mathrm{~mm}$ sciatic nerve gaps of rats were recorded. Liu et al. [11] developed an extrusion-based multi-nozzle additivelathe 3D bioprinting method to manufacture fully integrated bilayered nerve conduits. The bone marrow mesenchymal stem cells (BMSCs) laden inner layer was designed to provide an appropriate microenvironment, while the outer layer with good mechanical properties was designed for structural support. In vitro experiments showed that the proliferation and neurite outgrowth of PC12 cells were significantly improved in the BMSC embedded bilayered nerve conduits.

\section{Conclusions and perspectives}

The 3D bioprinting approach has garnered increased attention for providing nerve conduits with 3D complex and customized spatial structures to enhance the regeneration efficacy of peripheral nerves and is a promising biofabrication method. A number of critical limitations still need to be addressed if nerve conduits are to be successfully applied, especially for the repair of PNI with large defects $(>3 \mathrm{~cm})$. Firstly, more biochemical cues, including biomolecules and support cells, have to be incorporated in 3D bioprinting. But the fact is that very few bioinks simultaneously possess excellent printability, as well as mechanical and biological properties. Multi-material or multi-cell bioprinting is believed to be an effective solution to combine different biochemical/biological cues in one biofabrication process of nerve conduits, but how to integrate these factors, maximizing their features, and selecting suitable materials is a huge challenge. Secondly, current single 3D bioprinting method always exists a trade-off between printability and cell viability, so there is a trend toward the combination of multiple printing technologies to achieve a high-precision, high-complexity, and high-cell viability manufacturing of nerve conduits. Thirdly, the patient-specific nerve conduit has to be printed, which includes printing patient-derived cells into nerve conduits as the support cells and obtaining the geometrical information of PNI defects by scanning and modeling before printing, to realize the customization of nerve guide conduits.

Author contributions $\mathrm{YH}$ was involved in investigation and writingoriginal draft; JY contributed to conceptualization and writing-review and editing.

\section{Declarations}

Conflict of interest The authors declare that there is no conflict of interest.

Ethical approval This article does not contain any studies with human or animal subjects performed by any of the authors.

\section{References}

1. Nerve repair and regeneration market by products (nerve conduits, nerve wraps, vagus nerve stimulation, sacral nerve stimulation, spinal cord stimulation, TENS, TMS), application (neurorrhaphy, nerve grafting, stem cell therapy)_-global forecast to 2025 . Retrieved June 19, 2021

2. Lackington WA, Ryan AJ, O'Brien FJ (2017) Advances in nerve guidance conduit-based therapeutics for peripheral nerve repair. 
ACS Biomater Sci Eng 3(7):1221-1235. https://doi.org/10.1021/ acsbiomaterials.6b00500

3. Gu XS, Ding F, Williams DF (2014) Neural tissue engineering options for peripheral nerve regeneration. Biomaterials 35(24):6143-6156. https://doi.org/10.1016/j.biomaterials.2014. 04.064

4. Kehoe S, Zhang XF, Boyd D (2012) FDA approved guidance conduits and wraps for peripheral nerve injury: a review of materials and efficacy. Injury 43(5):553-572. https://doi.org/10.1016/j. injury.2010.12.030

5. Radulescu D, Dhar S, Young CM et al (2007) Tissue engineering scaffolds for nerve regeneration manufactured by ink-jet technology. Mater Sci Eng C 27(3):534-539. https://doi.org/10.1016/j. msec.2006.05.050

6. Johnson BN, Lancaster KZ, Zhen GH et al (2015) 3D printed anatomical nerve regeneration pathways. Adv Funct Mater 25(39):6205-6217. https://doi.org/10.1002/adfm.201501760

7. Singh A, Asikainen S, Teotia AK et al (2018) Biomimetic photocurable three-dimensional printed nerve guidance channels with aligned cryomatrix lumen for peripheral nerve regeneration. ACS Appl Mater Interf 10(50):43327-43342. https://doi.org/10.1021/ acsami.8b11677

8. Zhu W, Tringale KR, Woller SA et al (2018) Rapid continuous 3D printing of customizable peripheral nerve guidance conduits. Mater Today 21(9):951-959. https://doi.org/10.1016/j.mattod. 2018.04.001

9. Tao J, Zhang JM, Du T et al (2019) Rapid 3D printing of functional nanoparticle-enhanced conduits for effective nerve repair. Acta Biomater 90:49-59. https://doi.org/10.1016/j.actbio.2019. 03.047

10. Fang JH, Hsu HH, Hsu RS et al (2020) 4D printing of stretchable nanocookie@conduit material hosting biocues and magnetoelectric stimulation for neurite sprouting. NPG Asia Mater 12(1):116. https://doi.org/10.1038/s41427-020-00244-1

11. Liu JY, Zhang B, Li L et al (2021) Additive-lathe 3D bioprinting of bilayered nerve conduits incorporated with supportive cells. Bioac Mater 6(1):219-229. https://doi.org/10.1016/j.bioactmat. 2020.08.010 